\title{
On Delay-limited Average Rate of HARQ-based Predictor Antenna Systems
}

\author{
Hao Guo, Student Member, IEEE, Behrooz Makki, Senior Member, IEEE, \\ Mohamed-Slim Alouini, Fellow, IEEE, and Tommy Svensson, Senior Member, IEEE
}

\begin{abstract}
Predictor antenna (PA) system is referred to as a system with two sets of antennas on the roof of a vehicle, where the PAs positioned in the front of the vehicle are used to predict the channel state observed by the receive antennas (RAs) that are aligned behind the PAs. In this work, we study the effect of spatial mismatch on the accuracy of channel state information estimation, and analyze the delay-constrained average rate of hybrid automatic repeat request (HARQ)-based PA systems. We consider variable-length incremental redundancy (INR) HARQ protocols, and derive a closed-form expression for the maximum average rate subject to a maximum delay constraint. Also, we study the effect of different parameters such as the vehicle speed, the antenna distance and the rate allocation on the system performance. The results indicate that, compared to the openloop case, the delay-limited average rate is considerably improved with the proposed PA and HARQ-based scheme.
\end{abstract}

Index Terms-Average rate, backhaul, channel state information (CSI), HARQ, integrated access and backhaul (IAB), Marcum $Q$-function, mobility, mobile relay, predictor antenna, spatial correlation.

\section{INTRODUCTION}

Upcoming 5G use cases include, e.g., intelligent transport systems, autonomous vehicle control, factory automation and providing coverage to vehicles in high-speed scenarios, which are mainly categorized into the class of ultra-reliable low-latency communication (URLLC) [1]. In these vehicle communication-based applications, channel estimation and prediction play key role in the system design and optimization schemes [2]. However, due to the mobility of the users, the typical channel estimation methods may be inaccurate as the position of the transmit/receive antennas may change quickly. For this reason, the predictor antenna (PA) concept has been proposed and evaluated in, e.g., [3]-[7]. Here, the PA setup refers to as a system where two sets of antennas are deployed on the roof of a vehicle, and the PA(s) positioned at the front of the vehicle are used to predict the channel observed by the receive antennas (RAs) that are positioned behind the PA(s).

Typical PA systems have two problems, namely, spatial mismatch and spectral efficiency compared to, e.g., a static multiple input multiple output (MIMO) system with as many antenna elements. Specifically, due to, e.g., speed change or feedback/processing delay, the RAs may not reach the same

H. Guo and T. Svensson are with the Department of Electrical Engineering, Chalmers University of Technology, 41296 Gothenburg, Sweden (email: hao.guo@chalmers.se; tommy.svensson@chalmers.se).

B. Makki is with Ericsson Research, 41756 Gothenburg, Sweden (email: behrooz.makki@ericsson.com).

M.-S. Alouini is with the King Abdullah University of Science and Technology, Thuwal 23955-6900, Saudi Arabia (e-mail: slim.alouini@kaust.edu.sa). spatial point as the PA when they receive data. This spatial mismatch problem has been studied in $[4]-[6]$ where different techniques are applied to compensate for this effect. Moreover, in the existing PA setups, the PA is used only for channel estimation but not for data transmission. This may lead to poor performance, in terms of spectral efficiency compared to a corresponding static MIMO system.

From another perspective, hybrid automatic repeat request (HARQ) is a well-known approach to improve the data transmission reliability and efficiency. The main idea of HARQ is to re-transmit the message that experienced poor channel conditions in order to reduce the outage probability $[8]-[10]$. As the PA system includes the feedback link, i.e., from the PA to the base station (BS), HARQ can be supported by the PA structure in the moving scenarios. That is, the RA could potentially adjust its transmit rate/power based on the feedback from the PA. In this way, it is expected that the joint implementation of the PA and the HARQ scheme can improve the system efficiency and reliability.

In this paper, we develop an HARQ-based scheme for delay-limited PA systems. The goal is to maximize the delayconstrained average rate in the presence of imperfect channel state information at the transmitter side (CSIT). We model the spatial mismatch of the PA system as an equivalent non-central Chi-squared channel, and perform rate adaptation analysis. Particularly, using the incremental redundancy (INR) protocol and variable-length coding, we derive closed-form expressions for the maximum delay-limited average rate as well as for the optimal rate allocation. Finally, we evaluate the effect of different parameters such as transmission delay, variablelength coding and the vehicle speed on the average rate.

As opposed to [3], [4], where numerical and test-bed based analysis are presented, in this work we model the PA system analytically, and design a HARQ-based PA scheme. Moreover, our problem setup is different from the ones in [5]-[7] because of the HARQ setup and variable-length coding. Finally, our discussions on the average rate of the HARQ-assisted PA systems with imperfect CSIT have not been presented before.

The analytical and simulation results show that, while a HARQ-assisted PA system leads to considerable performance improvement, compared to the open-loop case, the average rate is remarkably affected by the spacial mismatch and delay constraint. Also, for a broad range of vehicle speeds/delay constraints, the optimal average rate scales with signal-tonoise ratio (SNR) almost logarithmically. Finally, while spatial mismatch may reduce the accuracy of CSIT, it increases the spatial diversity. Thus, depending on the vehicle speed, it may 


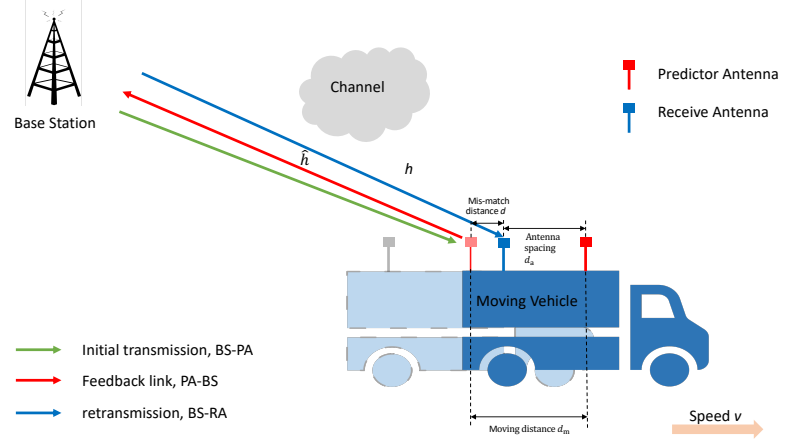

Fig. 1. HARQ-based PA system with spatial mismatch.

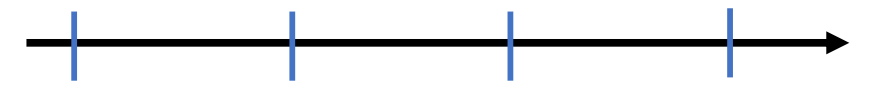

$t_{1}$ : BS sends data $t_{2}$ : PA 1 ) estimates $\widehat{H} \quad t_{3}$ : PA sends ACK or $t_{4}$ : If NACK, the BS and pilots to $P A$ from pilots; 2 NACK to the BS sends data to RA with initial rate $R$ generates ACK/NACK with adapted rate and power $p$

Fig. 2. Timing of the proposed PA-HARQ scheme.

improve or deteriorate the performance of PA-HARQ systems.

\section{SySTEM MODEL}

In the standard PA setup, proposed in $[3]-[6]$, the PA at the front of the vehicle is used only for channel estimation. As opposed, in this letter, we develop a setup where the PA is used for both channel estimation and initial data transmission. Our proposed setup and its timing can be seen in Figs. 1 and 2 respectively. Here, at $t_{1}$ the BS sends pilots as well as the encoded data with certain initial rate $R$ and fixed power $p$ to the PA. Then, at $t_{2}$, the PA estimates the BS-PA channel $\hat{h}$ from the received pilots. At the same time, the PA tries decoding the data and generates an acknowledgement (ACK) if the decoding of the data is successful. Otherwise, a negative acknowledgement (NACK) will be fed back. ACK or NACK is sent to the BS at $t_{3}$. Also, in case of a NACK, the PA also sends CSIT feedback to the BS so that it is aware of $\hat{h}$. Define $t_{3}-t_{1}=\delta$. Based on the received feedback, the BS can decide whether or not to retransmit the message. Finally, at $t_{4}$ and with NACK, the BS sends data to the RA with an adapted rate.

In this way, the signals received by the PA and the RA in the first and second rounds are

$$
\hat{y}=\sqrt{p} \hat{h} \hat{x}+z,
$$

and

$$
y=\sqrt{p} h x+z,
$$

respectively, where $p$ is the transmit power while $\hat{x}$ and $x$ are the transmitted message with unit variance, and $z \sim \mathcal{C N}(0,1)$ represents the independent and identically distributed (IID) complex Gaussian noise added at the receiver side. Also, $h$ represents the BS-RA channel coefficient. Note that, as explained in the following, the signals $\hat{x}$ and $x$ may be of different length, as a function of the channels quality and

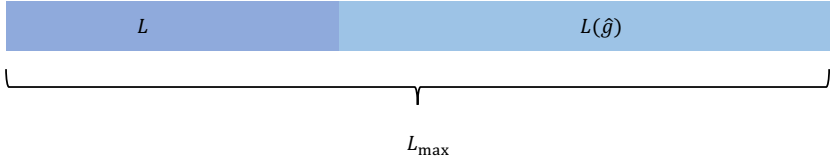

Fig. 3. Illustration of block length for two transmissions.

maximum delay constraint. Also, as presented in [5], the BSRA channel $h$ with spatial mismatch is modeled as [5, eq. (5)]

$$
h=\sqrt{1-\sigma^{2}} \hat{h}+\sigma q .
$$

Here, $\hat{h} \sim \mathcal{C N}(0,1)$ is assumed to be known at the BS, and $\sigma$ is a function of the mismatch distance $d$, which is defined as $d=\left|d_{\mathrm{m}}-d_{\mathrm{a}}\right|=\left|v \delta-d_{\mathrm{a}}\right|$. Here, $d_{\mathrm{m}}$ and $d_{\mathrm{a}}$ are defined as the moving distance with speed $v$ and time $\delta$, and the antenna separation between the PA and the RA, respectively, as illustrated in Fig. 1. Indeed, manipulate parameters such as $d_{\mathrm{m}}, d_{\mathrm{a}}$ and $v$ would end up with different $d$, leading to different level of spatial mismatch $\sigma$ with analytical derivations given in [6, Section III.A]. Also, we assume that $\sigma$ and $\hat{h}$ are known at the $\mathrm{BS}$, and $q \sim \mathcal{C N}(0,1)$ which is independent of the known channel value $\hat{h}$. In this way, the cumulative distribution function (CDF) of the channel gain, which is defined as $g=|h|^{2}$, for a given realization of $\hat{g}=|\hat{h}|^{2}$, can be described as

$$
F_{g \mid \hat{g}}(x)=1-\mathcal{Q}\left(\sqrt{\frac{2 \hat{g}\left(1-\sigma^{2}\right)}{\sigma^{2}}}, \sqrt{\frac{2 x}{\sigma^{2}}}\right) .
$$

Here,

$$
\mathcal{Q}(s, \rho)=\int_{\rho}^{\infty} x e^{-\frac{x^{2}+s^{2}}{2}} I_{0}(s x) \mathrm{d} x
$$

is the Marcum $Q$-function [11, eq. 1-5-2], where $s, \rho \geq 0$ and $I_{n}(x)=\left(\frac{x}{2}\right)^{n} \sum_{i=0}^{\infty} \frac{\left(\frac{x}{2}\right)^{2 i}}{i ! \Gamma(n+i+1)}$ is the $n$-order modified Bessel function of the first kind, and $\Gamma(z)=\int_{0}^{\infty} x^{z-1} e^{-x} \mathrm{~d} x$ represents the Gamma Function.

As shown in Fig. 3, let us denote the maximum acceptable delay constraint by $L_{\max }$ channel use (cu). Also, we present the block length allocated to the first and the second transmission signals by $L$ and $L(\hat{g})$, respectively. Here, $L$ is optimized based on average performance, and $L(\hat{g})$ is based on the instantaneous channel quality $\hat{g}$. This is because the initial transmission to the PA is performed based on no CSIT at the BS. However, in the second round, the transmission length is adapted based on the instantaneous channel quality $\hat{g}$ available at the BS. In the following, we optimize the average rate in the presence of variable-length coding and maximum delay constraint.

\section{Analytical Results}

Let us denote the initial rate by $R=\frac{K}{L}$ nats per channel use (npcu), where $K$ is the number of nats per codeword. Then, in the first transmission, if the instantaneous channel gain supports the rate, i.e., $R<\log (1+\hat{g} p)$ or equivalently, $\hat{g}>\frac{\theta}{p}$ where $\theta=e^{R}-1$, a ACK is sent back to the BS and the transmission stops. On the other hand, if $R>\log (1+\hat{g} p)$, 
an NACK as well as the instantaneous channel gain $\hat{g}$ are sent back to the BS. Then, in Round 2, we determine the required rate as

$$
R(\hat{g})=\log (1+\hat{g} p)=\frac{K}{L+L(\hat{g})},
$$

which is equivalent to

$$
L(\hat{g})=\frac{K}{\log (1+\hat{g} p)}-L .
$$

Let us denote $R_{\min }=\frac{K}{L_{\max }}$ which is limited by the delay constraint $L_{\max }$. If $L(\hat{g})+L>L_{\max }$ leading to $\hat{g}<\frac{e^{R_{\min }}-1}{p}$, no signal is sent in the second round because the maximum delay constraint can not be satisfied. Otherwise, if $\hat{g}>\frac{e^{R_{\min }}-1}{p}$, we send a redundancy sub-codeword with length $L(\hat{g})$.

In this way, the average rate, for given $\hat{g}$, is given by

$$
\eta \mid \hat{g}=R \mathcal{I}\left\{\hat{g}>\frac{\theta}{p}\right\}+\log (1+\hat{g} p) \mathcal{I}\left\{\frac{\theta_{\min }}{p} \leq \hat{g} \leq \frac{\theta}{p}, \mathcal{A}\right\} .
$$

Here, $\theta_{\min }$ is defined as $\theta_{\min }=e^{R_{\min }}-1$. Also, $\mathcal{I}(x)$ is the indicator function and $\mathcal{A}$ is the event that the message is decoded in the second round. Note that, with the rate allocation scheme of (6), it is straightforward to show that $\mathcal{A}$ occurs if and only if $g \geq \hat{g}$. In this way, (8) can be rewritten as

$\eta \mid \hat{g}=R \mathcal{I}\left\{\hat{g}>\frac{\theta}{p}\right\}+\log (1+\hat{g} p) \mathcal{I}\left\{\frac{\theta_{\min }}{p} \leq \hat{g} \leq \frac{\theta}{p}, g \geq \hat{g}\right\}$.

Finally, averaging the system performance over all possible $\hat{g}$, we can obtain the average rate as follows.

Theorem 1. The averaged rate of the proposed PA-HARQ scheme is approximately given by (10).

Proof. Using (9) and taking average over $\hat{g}$, whose probability density function (PDF) is given by $f_{\hat{g}}(x)=e^{-x}$, we have

$$
\begin{aligned}
\eta & =R \operatorname{Pr}\left(\hat{g} \geq \frac{\theta}{p}\right)+\int_{\theta_{\min } / p}^{\theta / p} \log (1+p x) f_{\hat{g}}(x) \operatorname{Pr}(\hat{g} \geq x) \mathrm{d} x \\
& =R e^{-\frac{\theta}{p}}+ \\
& \int_{\theta_{\min } / p}^{\theta / p} e^{-x} \log (1+p x) \mathcal{Q}\left(\sqrt{\frac{2\left(1-\sigma^{2}\right) x}{\sigma^{2}}}, \sqrt{\frac{2 x}{\sigma^{2}}}\right) \mathrm{d} x \\
& \stackrel{(b)}{=} R e^{-\frac{\theta}{p}}+ \\
& \int_{\theta_{\min } / p}^{\theta / p} e^{-x} \log (1+p x) \frac{1}{2}\left(1+e^{-\frac{2 x}{\sigma^{2}}} I_{0}\left(\frac{2 x}{\sigma^{2}}\right)\right) \mathrm{d} x \\
\stackrel{(c)}{\simeq} & R e^{-\frac{\theta}{p}}+\frac{1}{2} \log \left(1+\frac{\theta+\theta_{\min }}{2}\right) \times \\
& \int_{\theta_{\min } / p}^{\theta / p} e^{-x}\left(1+e^{-\frac{2 x}{\sigma^{2}}} \frac{e^{\frac{2 x}{\sigma^{2}}}}{\sqrt{2 \pi \frac{2 x}{\sigma^{2}}}}\right) \mathrm{d} x
\end{aligned}
$$

$$
\begin{aligned}
= & R e^{-\frac{\theta}{p}}+\frac{1}{2} \log \left(1+\frac{\theta+\theta_{\min }}{2}\right) \times \\
& \left(\int_{\theta_{\min } / p}^{\theta / p} e^{-x} \mathrm{~d} x+\int_{\theta_{\min } / p}^{\theta / p} \frac{e^{-x}}{\sqrt{2 \pi} \sqrt{\frac{2 x}{\sigma^{2}}}} \mathrm{~d} x\right) \\
\stackrel{(d)}{=} & R e^{-\frac{\theta}{p}}+\frac{1}{2} \log \left(1+\frac{\theta+\theta_{\min }}{2}\right)\left(\left(e^{-\frac{\theta_{\min }}{p}}-e^{-\frac{\theta}{p}}\right)+\right. \\
& \left.\frac{\sigma}{2}\left(\operatorname{erf}\left(\sqrt{\frac{\theta}{p}}\right)-\operatorname{erf}\left(\sqrt{\frac{\theta_{\min }}{p}}\right)\right)\right) .
\end{aligned}
$$

Here, $(b)$ is obtained by $\sqrt{\frac{2\left(1-\sigma^{2}\right) x}{\sigma^{2}}} \simeq \sqrt{\frac{2 x}{\sigma^{2}}}$ for small/moderate values of $\sigma$ as well as [11, Eq. (A-3-2)]

$$
\mathcal{Q}(x, x)=\frac{1}{2}\left(1+e^{-x^{2}} I_{0}\left(x^{2}\right)\right),
$$

and $(c)$ is obtained by using Taylor expansion for $\log (1+p x)$ at the point $x=\frac{\theta+\theta_{\min }}{2 p}$ and $I_{0}(x) \simeq \frac{e^{x}}{\sqrt{2 \pi x}}[12$, Eq. (9.7.1)]. Finally, $(d)$ uses the definition of the error function $\operatorname{erf}(x)=$ $\frac{2}{\sqrt{\pi}} \int_{0}^{x} e^{-t^{2}} \mathrm{~d} t$ and some manipulations.

Using (10), the optimal average rate can be found as $\eta_{\mathrm{opt}}=$ $\operatorname{argmax} \eta$, which is a one-dimensional equation and can be $R$

solved effectively. Finally, as a benchmark, the average rate of the open-loop setup, i.e., with no retransmissions, is given by

$$
\eta^{\text {Open-loop }}=\int_{\frac{\theta}{p}}^{\infty} R e^{-x} \mathrm{~d} x=R e^{-\frac{\theta}{p}}
$$

and the optimal rate allocation is found by setting the derivative of (12) with respect to $R$ equal to zero leading to $R=\mathcal{W}(p)$, where $\mathcal{W}(\cdot)$ represents the Lambert W-function $x e^{x}=y \Leftrightarrow x=\mathcal{W}(y)$ [13].

\section{Simulation Results}

In the simulations, we set $\delta=5 \mathrm{~ms}$, carrier frequency $f_{\mathrm{c}}=\frac{c}{\lambda}=2.68 \mathrm{GHz}$ with $\lambda$ being the carrier wavelength, and $d_{\mathrm{a}}=1.5 \lambda$, which affect the mismatch coefficient $\sigma$ as given in [6, Section III.A]. In Fig. 4, we demonstrate the optimal average rate in different cases for a broad range of SNRs which, because the noise has unit variance, we define as $10 \log _{10} P$. Here, $R_{\text {min }}$ is set to 2 and 3 npcu which corresponds to different delay constraint $L_{\max }$ (cf. Sec. III), and $\sigma=0.1,0.9$. The approximation values are obtained by Theorem 11 and the open-loop case is based on (12). Figure 5 presents optimal values of $R$ for different values of $R_{\text {min }}$ as a function of SNR. Here, we set $R_{\min }=2,3 \mathrm{npcu}$, and $\sigma=0.1$. Finally, the optimal average rate as a function of vehicle velocities is illustrated in Fig. 6, with $R_{\min }=2 \mathrm{npcu}$, and SNR set to 14,16 , and $18 \mathrm{~dB}$. We also present the approximation values from Theorem 1 . Here, as explained in [5. Section II], manipulating $v$ is equivalent to changing the level of spatial correlation $\sigma$ for given values of $\delta, f_{\mathrm{c}}$ and $d_{\mathrm{a}}$ in (3).

According to the figures, we can conclude the following:

- The approximation scheme of Theorem 1 is tight for a broad range of values of SNR (Figs. 46), $R_{\min }$ (Figs. 4 5) as well as $\sigma$, or equivalently, $v$ (Fig. 4. 6). Thus, for 


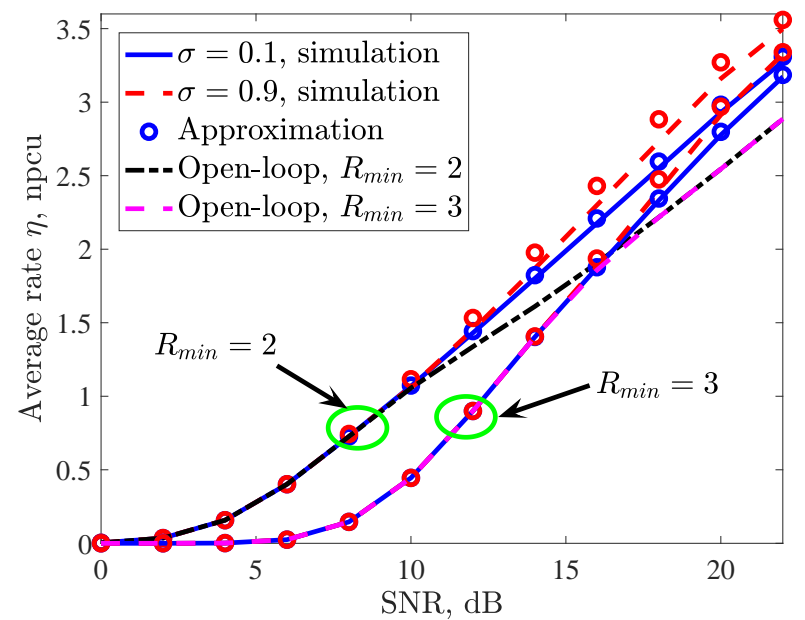

Fig. 4. $\eta_{\text {opt }}$ vs. SNR. $R_{\min }$ is set to 2 and 3 npcu, and $\sigma=0.1,0.9$.

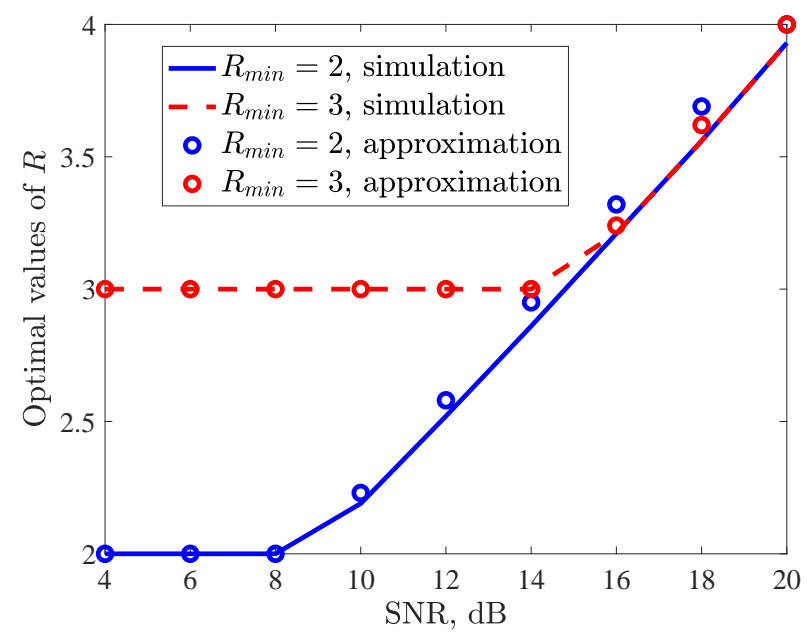

Fig. 5. Optimal values of $R$ vs. SNR, $R_{\min }=2,3 \mathrm{npcu}$, and $\sigma=0.1$.

different parameter settings, the average rate optimization problem for the HARQ-based PA system can be well determined by Theorem 1 .

- With deployment of the PA-HARQ, remarkable average rate gain is achieved, compared to open-loop, especially in moderate/high SNRs (Fig. 4). Also, the average rate increases when the spatial correlation between the PA and the RA is low, i.e., when $\sigma$ increases (Figs. 4, 6).

- As can be seen from Fig. 5, for a broad range of parameter settings and SNRs, the optimal rate $R$ increases with SNR, in the log domain, almost linearly. Also, as $R_{\min }$ increases, i.e., with more stringent delay-constraint, higher initial transmission rate $R$ is required for the optimal average rate, as can be seen from the flat regions at the beginning the figure.

- In Fig. 6, there is a minimum value for the average rate. This is intuitive because at low and high speed, i.e., $v<$ $110 \mathrm{~km} / \mathrm{h}$ and $v>125 \mathrm{~km} / \mathrm{h}$, we have larger $\sigma$, which although it reduces the CSIT accuracy, provides higher average rate since it gives better spatial diversity of the channel. As a result, the performance of HARQ protocol is improved. The lowest value of $\eta$ is observed at around $120 \mathrm{~km} / \mathrm{h}$, which matches our straightforward calculation from $d=\left|d_{\mathrm{m}}-d_{\mathrm{a}}\right|$.

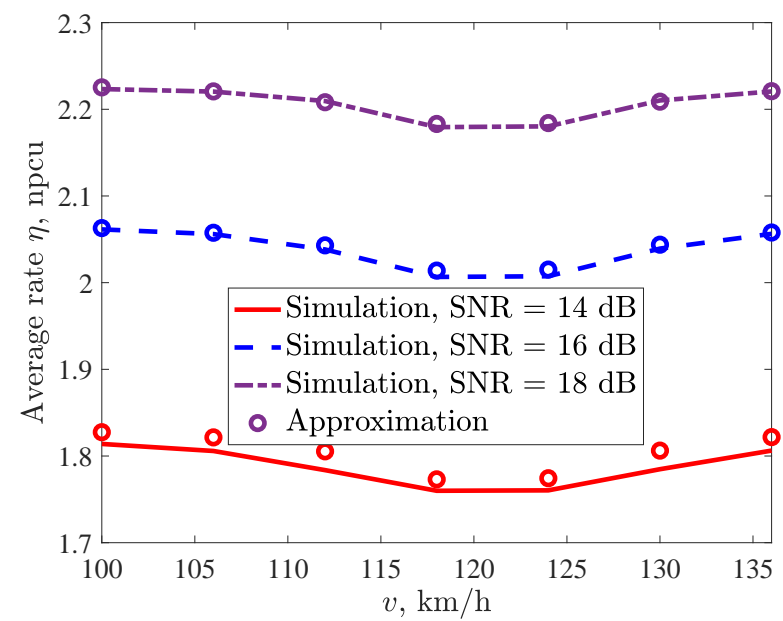

Fig. 6. $\eta_{\text {opt }}$ vs. $v$. $R_{\min }=2 \mathrm{npcu}$, and $\mathrm{SNR}=14,16,18 \mathrm{~dB}$.

\section{CONCLUSION}

We proposed an HARQ-based approach in order to compensate for the spatial mismatch and spectral under-utilization problems of PA systems. We designed the system such that the feedback from the PA can be used to adjust the codeword length in order to improve the average rate. The simulation and analytical results show that, while HARQ-assisted PA system leads to considerable performance improvement, compared to open-loop communications, the average rate is remarkably affected by the spacial mismatch, transmitted SNR as well as the delay constraint.

\section{REFERENCES}

[1] S. Parkvall, E. Dahlman, A. Furuskar, and M. Frenne, "NR: The new 5G radio access technology," IEEE Commun. Standards Mag., vol. 1, no. 4, pp. 24-30, Dec. 2017.

[2] L. Liang, H. Peng, G. Y. Li, and X. Shen, "Vehicular communications: A physical layer perspective," IEEE Trans. Veh. Technol., vol. 66, no. 12 , pp. 10647-10659, Dec. 2017.

[3] M. Sternad, M. Grieger, R. Apelfröjd, T. Svensson, D. Aronsson, and A. B. Martinez, "Using predictor antennas for long-range prediction of fast fading for moving relays," in Proc. IEEE WCNCW, Paris, France, Apr. 2012, pp. 253-257.

[4] D.-T. Phan-Huy, M. Sternad, and T. Svensson, "Making 5G adaptive antennas work for very fast moving vehicles," IEEE Intell. Transp. Syst. Mag., vol. 7, no. 2, pp. 71-84, Apr. 2015.

[5] H. Guo, B. Makki, and T. Svensson, "Rate adaptation in predictor antenna systems," IEEE Wireless. Commun. Lett., Dec. 2019.

[6] H. Guo, B. Makki, M.-S. Alouini, and T. Svensson, "A semilinear approximation of the first-order Marcum $Q$-function with application to predictor antenna systems," Jan. 2020, available at: https://arxiv.org/abs/2001.09264.

[7] —- "Power allocation in HARQ-based predictor antenna systems," Apr. 2020, available at http://arxiv.org

[8] B. Makki, T. Svensson, and M. Zorzi, "Finite block-length analysis of the incremental redundancy HARQ," IEEE Wireless. Commun. Lett., vol. 3, no. 5, pp. 529-532, Oct. 2014.

[9] G. Caire and D. Tuninetti, "The throughput of hybrid-ARQ protocols for the Gaussian collision channel," IEEE Trans. Inf. Theory, vol. 47, no. 5, pp. 1971-1988, Jul. 2001.

[10] S. Sesia, G. Caire, and G. Vivier, "Incremental redundancy hybrid ARQ schemes based on low-density parity-check codes," IEEE Trans. Commun., vol. 52, no. 8, pp. 1311-1321, Aug. 2004.

[11] M. Schwartz, W. R. Bennett, and S. Stein, Communication Systems and Techniques. John Wiley \& Sons, 1995.

[12] M. S. Abramowitz and I. Stegun, "Handbook of Mathematical Functions with Formulas, Graphs, and Mathematical Tables," New York: Dover, 1972.

[13] R. M. Corless, G. H. Gonnet, D. E. Hare, D. J. Jeffrey, and D. E Knuth, "On the Lambert $\mathcal{W}$ function," Advances in Computational Mathematics, vol. 5, no. 1, pp. 329-359, Dec. 1996. 